\title{
Raster-scan imaging with normal-incidence, midinfrared InAs/GaAs quantum dot infrared photodetectors
}

\author{
A. D. Stiff-Roberts, S. Chakrabarti, S. Pradhan, B. Kochman, and P. Bhattacharyaa) \\ Solid State Electronics Laboratory, Department of Electrical Engineering and Computer Science, \\ University of Michigan, Ann Arbor, Michigan 48109-2122
}

(Received 18 January 2002; accepted for publication 5 March 2002)

\begin{abstract}
We demonstrate normal incidence infrared imaging with quantum dot infrared photodetectors using a raster-scan technique. The device heterostructure, containing multiple layers of InAs/GaAs self-organized quantum dots, were grown by molecular-beam epitaxy. Individual devices have been operated at temperatures as high as $150 \mathrm{~K}$ and, at $100 \mathrm{~K}$, are characterized by $\lambda_{\text {peak }}=3.72 \mu \mathrm{m}$, $J_{\text {dark }}=6 \times 10^{-10} \mathrm{~A} / \mathrm{cm}^{2}$ for a bias of $0.1 \mathrm{~V}$, and $D^{*}=2.94 \times 10^{9} \mathrm{~cm} \mathrm{~Hz}^{1 / 2} / \mathrm{W}$ at a bias of $0.2 \mathrm{~V}$. Raster-scan images of heated objects and infrared light sources were obtained with a small (13 $\times 13$ ) interconnected array of detectors (to increase the photocurrent) at $80 \mathrm{~K}$. (C) 2002 American Institute of Physics. [DOI: 10.1063/1.1476387]
\end{abstract}

Infrared detection and imaging in the midwavelength infrared (MWIR) $(3-5 \mu \mathrm{m})$ and long-wavelength infrared (LWIR) $(8-14 \mu \mathrm{m})$ ranges are crucial for a variety of applications, ranging from military night vision to environmental monitoring. Quantum dot infrared photodetectors (QDIPs), made with self-organized $\mathrm{In}(\mathrm{Ga}) \mathrm{As} / \mathrm{Ga}(\mathrm{Al})$ As quantum dots have recently demonstrated very promising results in terms of mid- and far-infrared detection. ${ }^{1-8}$ These devices offer three favorable attributes: (i) they are inherently sensitive to normally incident infrared light, (ii) they can potentially achieve high-temperature operation $(>100 \mathrm{~K})$ due to an increased responsivity resulting from the phonon bottleneck and large lifetime in the excited energy levels of the quantum dot, ${ }^{9,10}$ and (iii) QDIPs have a low dark current because of the three-dimensional quantum confinement of carriers inside the dot, which results in the equality of the photoionization energy and the thermionic emission energy. ${ }^{11}$ We have recently reported a normal-incidence, vertical InAs/GaAs QDIP with a single- $\mathrm{Al}_{0.3} \mathrm{Ga}_{0.7} \mathrm{As}$ current-blocking barrier that yielded a specific detectivity, or $D^{*}$, of approximately 3 $\times 10^{9} \mathrm{~cm} \mathrm{~Hz}^{1 / 2} / \mathrm{W}$ at a temperature of $100 \mathrm{~K}$. We were also able to operate (signal-to-noise ratio, $\mathrm{S} / \mathrm{N}$, greater than unity) this device at a temperature as high as $150 \mathrm{~K}$. However, to date there has been no demonstration of infrared imaging with QDIPs.

The design and fabrication of a fully operational, largearea focal plane array (FPA) is both expensive and timeconsuming, due in large part to the silicon read-out circuit that must be hybrid bump bonded (or flip-chip bonded) to the detector array. ${ }^{12}$ Such a read-out circuit allows for independent access to each pixel in the detector array for digital signal processing of an image. In an effort to demonstrate imaging with QDIPs in a less rigorous manner, we have developed a raster-scan imaging system in which the field-ofview is scanned simultaneously in the $x$ and $y$ axes by a raster-scan motion provided by two mirrors. An array of photocurrent/voltage values is compiled as the field-of-view array elements are scanned either by a single detector or a

${ }^{a)}$ Electronic mail: pkb@eecs.umich.edu small interconnected detector array. In this letter, we demonstrate infrared imaging with self-organized InAs/GaAs quantum dot infrared photodetectors using a raster scanning technique.

The InAs/GaAs quantum dot infrared photodetector heterostructures were grown by solid-source molecular-beam epitaxy using a Varian GEN-II machine with an uncracked $\mathrm{As}_{4}$ source. A $0.5 \mu \mathrm{m}$ silicon-doped $\left(n=2 \times 10^{18} \mathrm{~cm}^{-3}\right)$ GaAs contact layer was deposited on a semi-insulating (100) GaAs substrate. Subsequently, ten layers of 2.2 monolayer InAs quantum dots, surrounded by $250 \AA \mathrm{GaAs}$ barriers, were deposited. A $400 \AA$ intrinsic $\mathrm{Al}_{0.3} \mathrm{Ga}_{0.7} \mathrm{As}$ currentblocking barrier $^{8}$ was grown next, and finally, a $0.1 \mu \mathrm{m}$ silicon-doped $\left(n=2 \times 10^{18} \mathrm{~cm}^{-3}\right)$ GaAs top contact layer was grown. This device heterostructure is the same as that used in our high-detectivity, high-operating temperature QDIPs discussed in detail elsewhere, ${ }^{7,8}$ and it is shown schematically in the inset to Fig. 1(a).

We have previously characterized QDIPs made from the heterostructure just described, and it was chosen for the present imaging experiment because of the promising performance characteristics of the devices. ${ }^{7,8}$ The area exposed to IR radiation for a typical device is $2.83 \times 10^{5} \mu \mathrm{m}^{2}$. At a detector temperature of $100 \mathrm{~K}$, the dark-current density was as low as $6 \times 10^{-10} \mathrm{~A} / \mathrm{cm}^{2}$ at a bias voltage of $0.1 \mathrm{~V}$. The spectral response, measured at $78 \mathrm{~K}$, yielded a peak wavelength, $\lambda_{\text {peak }}$ of $3.72 \mu \mathrm{m}$, and a spectral width, $\Delta \lambda / \lambda_{\text {peak }}$, equal to 0.3 , which is fairly broad. The peak responsivity, $R_{\text {peak }}$, was $2 \mathrm{~mA} / \mathrm{W}$ for a bias of $0.3 \mathrm{~V}$ at $100 \mathrm{~K}$. This low responsivity results from the AlGaAs barrier, which not only blocks the dark current, but the photocurrent as well. Despite the low $R_{\text {peak }}$, a maximum specific detectivity, $D^{*}$, of 2.94 $\times 10^{9} \mathrm{~cm} \mathrm{~Hz}^{1 / 2} / \mathrm{W}$ is obtained at a bias of $0.2 \mathrm{~V}$ and a detector temperature of $100 \mathrm{~K}$. Also, the device was able to operate at a temperature as high as $150 \mathrm{~K}$, that is, the $\mathrm{S} / \mathrm{N}>1$ as measured by a fast Fourier transform analyzer. The darkcurrent density at a temperature of $150 \mathrm{~K}$ and a bias of $0.1 \mathrm{~V}$ is $1.07 \times 10^{-5} \mathrm{~A} / \mathrm{cm}^{2}$. The bias-dependent detectivities of a typical device at $100 \mathrm{~K}$ are illustrated in Fig. 1(a).

While raster-scan imaging should work with a single de- 


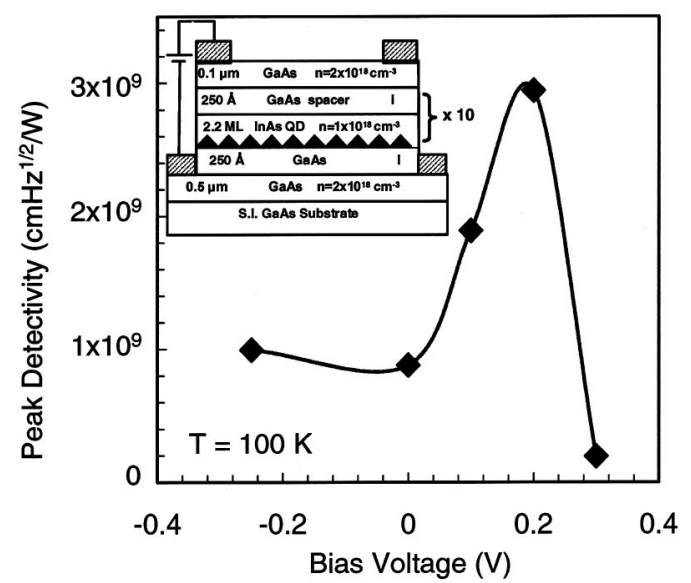

(a)

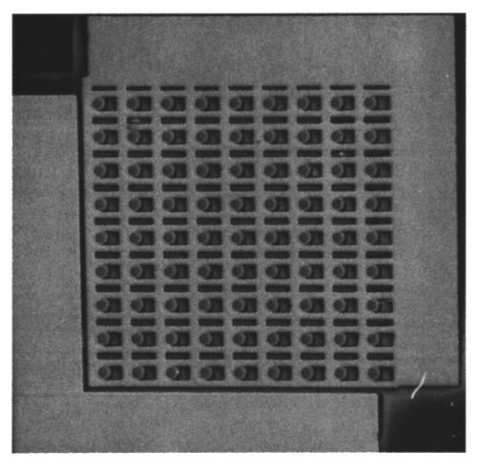

(b)

FIG. 1. (a) Typical specific detectivity $\left(D^{*}\right)$ as a function of bias voltage for the ten-layer InAs/GaAs QDIP heterostructure shown schematically in the inset, and (b) scanning electron microscopy image of a $(9 \times 9)$ interconnected, nonaddressable InAs/GaAs QDIP array with $40 \mu \mathrm{m}$ mesa size and $120 \mu \mathrm{m}$ pitch.

tector, a small array was actually used because it is easier to collect infrared light over a larger area. Also, the average photocurrent from an array should be larger, and therefore, much easier to distinguish from background noise. Standard photolithography and wet-etch processing were used to fabricate $(4 \times 4),(9 \times 9)$, and $(13 \times 13)$ individually addressable and nonaddressable (interconnected) arrays of vertical, mesa-shaped QDIPs. The pixel diameter was $40 \mu \mathrm{m}$, and the array pitch was $120 \mu \mathrm{m}$. The photomicrograph of an interconnected, $(9 \times 9)$ QDIP array is shown in Fig. 1(b). The entire array has a single-top contact and a single-bottom contact for all of the pixels, effectively behaving as a single detector with a very large optical area and generating a single-photocurrent signal. In order to assess the uniformity of the dots and device processing, we measured the roomtemperature dark current of the devices in an individually addressed $(4 \times 4)$ array. The result is shown in Fig. 2(a). It is apparent that the dark current is fairly uniform (for $0.1 \mathrm{~V}$ bias, the standard deviation, $\sigma$, is $3.07 \times 10^{-5}$ ) across this small array despite the large inhomogeneity that is characteristic of the self-organized growth mode. However, it is obvious that FPAs will involve larger areas on the grown wafer.

Raster scanning of the field of view (object to be imaged or infrared source) was accomplished using two gold-plated mirrors (highly reflective in the MWIR and LWIR ranges) servoactuated by galvanometers and mounted in an $X Y$ bracket. User interfacing with the drive circuitry of the gal-

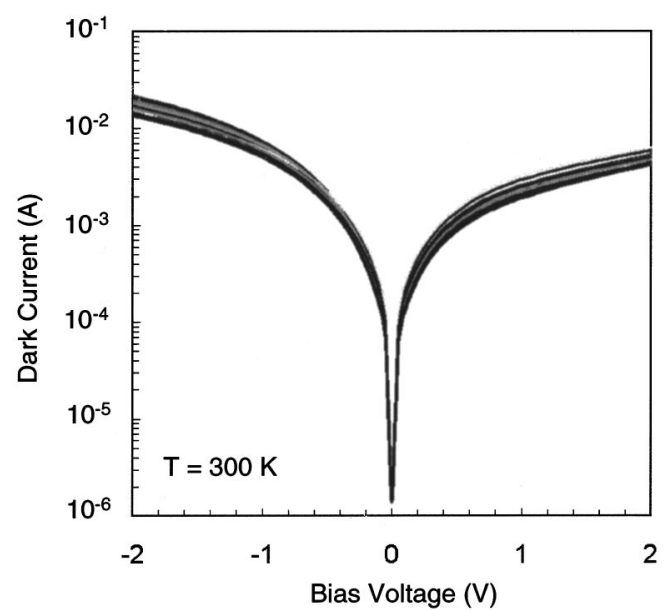

(a)

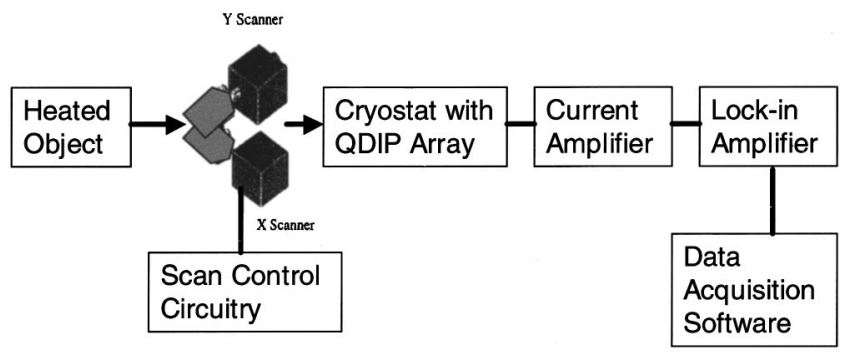

(b)

FIG. 2. (a) Room-temperature $(300 \mathrm{~K})$ dark current-voltage curves for each pixel in an addressable $4 \times 4$ QDIP array, and (b) schematic of the raster scan imaging system.

vanometer is achieved with a computer-interfaced scan controller. The raster-scan motion of the mirrors directs the infrared light onto the QDIP for detection. The $(13 \times 13)$ QDIP array, with which the imaging experiments were conducted, was housed in a cryostat with a KRS-5 entrance window and held at a temperature of $80 \mathrm{~K}$. The photocurrent produced in the QDIP array is amplified by a low-noise current amplifier, which also provides the bias to the array. The output is further amplified by lock-in techniques, and the amplified signal is transmitted to the data acquisition system wherein each photocurrent/voltage signal obtained from the QDIP corresponds to the raster-scan mirror positions and hence to a segment of the field of view. A schematic of the raster-scan system is shown in Fig. 2(b). The resolution of images obtained by this raster scan technique is determined by the incremental motion of the two scanning mirrors, as well as the integration time (time constant) of the lock-in amplifier. In other words, small steps taken by the galvanometers controlling the scanning mirrors correspond to a high resolution of the image. Based on the images we have obtained, which are discussed next, we estimate that our raster-scan measurement system has a resolution of approximately $0.1 \mathrm{~cm}$ in object feature size.

Ideally, infrared detectors are used to image easily recognizable scenes or objects that are warm or hot. However, in our measurement system, a size constraint was imposed by the scanning mirrors in that the exit aperture in the $X Y$ bracket limited the field of view of the mirrors. Therefore, we have imaged portions of heated objects or the illumination from suitable light sources. All of the objects imaged were placed directly in front of the $X Y$ mirror bracket, at $90^{\circ}$ 


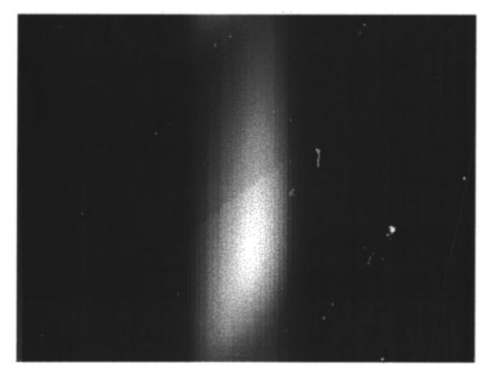

(a)

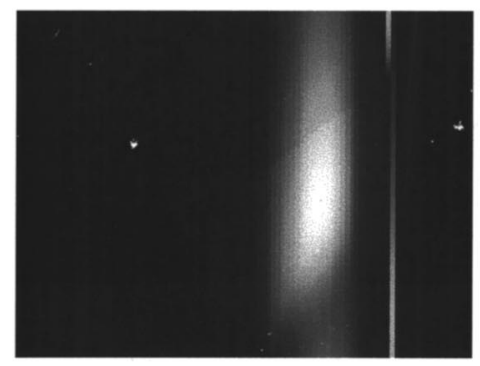

(b)

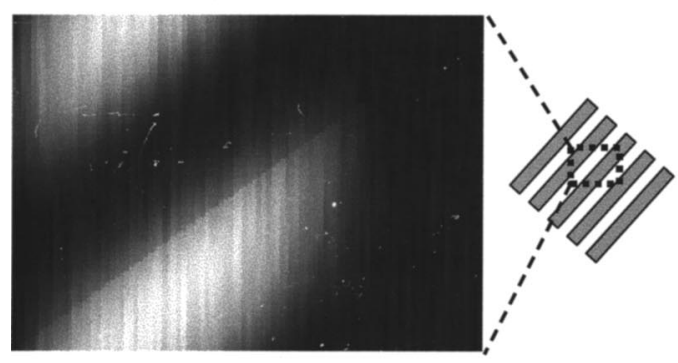

(c)

FIG. 3. Raster-scanned images obtained from the $(13 \times 13)$ QDIP array at $80 \mathrm{~K}$ : (a) $20 \mathrm{~W}$ broadband infrared globar source through a circular pinhole, (b) $700{ }^{\circ} \mathrm{C}$ graphite furnace igniter through a circular hole and linear slit, and (c) $500^{\circ} \mathrm{C}$ heating element from a hot plate, shown schematically in the inset, partially showing two metal strips.

to the QDIP array and no more than $40 \mathrm{~cm}$ away from the mirror bracket entrance window. Figure 3(a) shows the image of a $20 \mathrm{~W}$ broadband infrared globar source through a circular pinhole with a diameter of approximately $2 \mathrm{~cm}$. Figure 3(b) is the image of a $700{ }^{\circ} \mathrm{C}$ furnace igniter shielded by an aluminum block with a $1 \mathrm{~cm}$ diameter circular pinhole and a linear slit. There is some distortion present because the computer program used to display the images treats each array element as a rectangle, whereas the image array is actually square. Figure 3(c) depicts the partial image, in this case limited by the field of view of the scanning mirrors, of the heating element of a hot plate at $500{ }^{\circ} \mathrm{C}$. Portions of the heated strips [schematically shown in the inset of Fig. 3(c)] are seen as the bright regions. The width of each metal strip is $2 \mathrm{~cm}$ with a $1.5 \mathrm{~cm}$ space between strips. All three images were obtained at different distances from the QDIP array, thus size comparisons cannot be made from image to image. Experiments are in progress to demonstrate imaging of larger objects at higher temperatures. These results indicate that, in spite of the low responsivity of the QDIPs, they can be used for imaging. With progressive improvement of device performance, they should be applicable to FPAs.

In conclusion, through the use of a raster-scan imaging system, we have been able to demonstrate infrared imaging with QDIPs. Such a demonstration indicates that QDIPs are a viable option for MWIR and LWIR detection at elevated temperatures. With improvement in the responsivity of the devices, the fabrication of FPAs can be attempted.

The authors would like to acknowledge Steve Kennerly and Dr. John Little at the U.S. Army Research Laboratory in Adelphi, MD, for their suggestions, as well as Professor Sanjay Krishna from the Center for High Technology Materials at the University of New Mexico in Albuquerque, for valuable discussions. This work is supported by DARPA under Grant No. DAAD19-00-1-0394.

${ }^{1}$ K. W. Berryman, S. A. Lyon, and M. Segev, Appl. Phys. Lett. 70, 1861 (1997).

${ }^{2}$ J. Phillips, K. Kamath, and P. Bhattacharya, Appl. Phys. Lett. 72, 2020 (1998).

${ }^{3}$ S. Maimon, E. Finkman, and G. Bahir, Appl. Phys. Lett. 73, 2003 (1998).

${ }^{4}$ D. Pan, E. Towe, and S. Kennerly, Appl. Phys. Lett. 73, 1937 (1998).

${ }^{5}$ J. Phillips, P. Bhattacharya, S. W. Kennerly, D. W. Beekman, and M. Dutta, IEEE J. Quantum Electron. 35, 936 (1999).

${ }^{6}$ S. Y. Wang, S. D. Lin, H. W. Wu, and C. P. Lee, Appl. Phys. Lett. 78, 1023 (2001).

${ }^{7}$ A. D. Stiff, S. Krishna, P. Bhattacharya, and S. Kennerly, Appl. Phys. Lett. 79, 21 (2001).

${ }^{8}$ A. D. Stiff, S. Krishna, P. Bhattacharya, and S. Kennerly, IEEE J. Quantum Electron. 37, 1412 (2001).

${ }^{9}$ D. Klotzkin, K. Kamath, and P. Bhattacharya, IEEE Photonics Technol. Lett. 9, 1301 (1997).

${ }^{10}$ J. Urayama, T. B. Norris, J. Singh, and P. Bhattacharya, Phys. Rev. Lett. 86, 4930 (2001).

${ }^{11}$ V. Ryzhii, Semicond. Sci. Technol. 11, 759 (1996).

${ }^{12}$ H. C. Liu, M. Buchanan, J. Li, Z. R. Wasilewski, P. H. Wilson, P. A. Marshall, R. A. Barber, P. Chow-Chong, J. W. Fraser, and J. Stapledon, Applications of Photonic Technology 2, edited by G. A. Lampropoulos and R. A. Lessard (Plenum, New York, 1997), pp. 311-318. 